\title{
A nova re-accretion model for J-type carbon stars ${ }^{\star}$
}

\author{
S. Sengupta, R. G. Izzard, and H. H. B. Lau
}

\author{
Argelander-Institut für Astronomie, Auf dem Hügel 71, 53121 Bonn, Germany \\ e-mail: sutirtha@astro.uni-bonn.de
}

Received 1 August 2013 / Accepted 3 October 2013

\begin{abstract}
The J-type carbon (J)-stars constitute 10-15\% of the observed carbon stars in both our Galaxy and the Large Magellanic Cloud (LMC). They are characterized by strong ${ }^{13} \mathrm{C}$ absorption bands with low ${ }^{12} \mathrm{C} /{ }^{13} \mathrm{C}$ ratios along with other chemical signatures peculiar for typical carbon stars, e.g. a lack of s-process enhancement. Most of the J-stars are dimmer than the N-type carbon stars some of which, by hot-bottom burning, make ${ }^{13} \mathrm{C}$ only in a narrow range of masses. We investigate a binary-star formation channel for $\mathrm{J}$-stars involving re-accretion of carbon-rich nova ejecta on main-sequence companions to low-mass carbon-oxygen white-dwarfs. The subsequent evolution of the companion stars in such systems is studied with a rapid binary evolutionary code to predict chemical signatures of nova pollution in systems which merge into giant single stars. A detailed population synthesis study is performed to estimate the number of these mergers and compare their properties with observed J-stars. Our results predict that such nova polluted mergers evolve with low luminosities as well as low ${ }^{12} \mathrm{C} /{ }^{13} \mathrm{C}$ ratios like the majority of observed J-stars (e.g. in the LMC) but cannot account for the observed fraction of J-stars in existing surveys of carbon stars.
\end{abstract}

Key words. stars: carbon - stars: chemically peculiar - novae, cataclysmic variables - white dwarfs - stars: abundances stars: statistics

\section{Introduction}

Stars spend about $90 \%$ of their lifetime on the main sequence, during which they convert hydrogen $(\mathrm{H})$ to helium $(\mathrm{He})$ in their cores by the pp-chain or the CNO-cycle. Following core hydrogen exhaustion they leave the main sequence and depending on their mass, many proceed to advanced stages of nuclear burning. In low and intermediate-mass stars $\left(M \sim 0.8-8 M_{\odot}\right)$, the triple- $\alpha$ reaction acts as a source of energy after depletion of internal hydrogen and produces carbon during their He-burning phase of evolution. When these stars ascend the asymptotic giant branch (AGB), the products of He-burning (mainly ${ }^{12} \mathrm{C}$ ) along with s-process elements formed through neutron captures are mixed into the convective envelope during the thermally pulsing (TP) phase and brought to the stellar surface by third dredge-up (TDU). This AGB scenario explains the origin of the majority of all carbon $(\mathrm{C})$-stars (characterized by surface $\mathrm{C} / \mathrm{O}>1$ by number) - the N-type stars (Iben \& Renzini 1983). However, spectral classification of C-stars reveals other peculiar scenarios of carbon enrichment especially in binaries (Wallerstein \& Knapp 1998). Mass transfer from a carbon-rich AGB primary star can pollute its binary companion with enough carbon to turn it into a dwarf carbon star which explains the origin of the $\mathrm{CH}$ and Ba-stars (Jorissen et al. 1998). In this work, we investigate a binary model that aims to explain the origin of possibly the least understood class among all C-stars - the J-type (J)-stars - whose evolutionary origin has remained a mystery for decades.

\subsection{J-stars}

The J-stars are a class of red-giant $\mathrm{C}$-stars that are characterized by strong ${ }^{13} \mathrm{C}$ bands (Bouigue 1954 ) and low ${ }^{12} \mathrm{C} /{ }^{13} \mathrm{C}$ ratios (Ohnaka \& Tsuji 1999), and constitute a significant fraction

$\star$ Appendices A and B are available in electronic form at http://www. aanda.org
(10-15\%) of all carbon stars in our Galaxy (Abia \& Isern 2000) and the Large Magellanic Cloud (LMC; Morgan et al. 2003). AGB models of C-stars cannot explain most of the chemical peculiarities associated with J-stars e.g. no s-process overabundance and Li enhancements (Hatzidimitriou et al. 2003). Moreover, their luminosities and variability classes indicate that they are less evolved objects than the N-type C-stars (Abia \& Isern 2000). However, the presence of high luminosity J-stars in our Galaxy (e.g. WZ Cas) suggests the existence of at least two types of J-stars, with different formation scenarios. Moreover, an intriguing sub-class of J-stars known as silicate carbon stars, characterized by oxygen-rich circumstellar material possibly stored in a circumbinary disk (Lloyd Evans 1991), points to a binary origin for this peculiar class of C-stars (e.g. BM-Gem; Ohnaka et al. 2008; Izumiura et al. 2008). A recent study exploring white-dwarf (WD) red-giant (RG) binary mergers in the context of the R-type C-stars, has found a possible evolutionary channel for J-stars (Zhang \& Jeffery 2013). We investigate another binary scenario considering chemical pollution of mainsequence (MS) companions to WDs in classical novae explosions and study their long-term evolutionary outcome in context of peculiarities associated with J-stars.

\section{2. ${ }^{13} \mathrm{C}$ enhancement scenarios}

The triple- $\alpha$ process operating in cores of AGB stars makes ${ }^{12} \mathrm{C}$ which is subsequently brought to their surface during dredge up thus forming a C-star. The synthesis of ${ }^{13} \mathrm{C}$ requires a further proton capture on ${ }^{12} \mathrm{C}$ that can only occur in regions with conditions suitable for operation of the $\mathrm{CNO}$ cycle. The $\beta$-unstable isotope ${ }^{13} \mathrm{~N}$ produced in $\mathrm{CN}$ cycle by ${ }^{12} \mathrm{C}(\mathrm{p}, \gamma){ }^{13} \mathrm{~N}$ decays to ${ }^{13} \mathrm{C}$ which when transported to the stellar surface leads to low ${ }^{12} \mathrm{C} /{ }^{13} \mathrm{C}$ ratios characteristic of J-stars. Such processes occur during the evolutionary stages of both single and binary stellar systems as described next. 


\subsubsection{Single stars}

It is not easy to construct a model of an AGB star with the peculiar properties of J-stars. AGB star models predict low ${ }^{12} \mathrm{C} /{ }^{13} \mathrm{C}$ ratios for masses $M>4 M_{\odot}$ if temperatures at the base of the convective envelope are sufficiently high for hot bottom burning (HBB, Boothroyd et al. 1995). However, the CN-cycle operating under such conditions destroys ${ }^{12} \mathrm{C}$ and consequently lowers the $\mathrm{C} / \mathrm{O}$ ratio in the envelope to below 1 making the star O-rich. Only narrow ranges of masses and mass-loss rates lead to short phases $(\sim 0.1 \mathrm{Myr})$ on the AGB with both $\mathrm{C} / \mathrm{O}>1$ and ${ }^{12} \mathrm{C} /{ }^{13} \mathrm{C}<10$ corresponding to J-stars. Moreover, TDU in the TPAGB phase also enriches of the envelope with s-process elements that are synthesized in the inter-pulse period when ${ }^{13} \mathrm{C}$ formed in the intershell region acts as a neutron source via the ${ }^{13} \mathrm{C}(\alpha, n){ }^{16} \mathrm{O}$ reaction (Straniero et al. 1995). However, observational studies of J-stars show that abundances of s-process elements with respect to iron for most of them are nearly solar (e.g. Utsumi 1985). Later works also conclude that mean heavy element abundance among the J-stars in their sample is compatible with no enrichment (Abia \& Isern 2000).

Statistically the narrow range of masses for which the HBB AGB models predict such chemical features cannot account for the observed fraction of J-stars among C-stars . Luminosity estimates from carbon-star surveys in the LMC also suggest that the HBB AGB models cannot explain the dimmer $\left(M_{\mathrm{Bol}}>-5\right)$ majority of J-stars (Hatzidimitriou et al. 2003).

\subsubsection{Binary systems}

While the HBB AGB scenario explains qualitatively the luminous J stars, a different chain of events seems to be responsible for the anomalous features observed in the majority of J-stars. Some other classes of C-stars also show traits common with J-stars e.g. the $\mathrm{R}$ and $\mathrm{CH}$ stars which also show low ${ }^{12} \mathrm{C} /{ }^{13} \mathrm{C}$ ratios (Vanture 1992) and are associated with binary formation scenarios (e.g. Izzard et al. 2007). Moreover observations of silicate carbon stars which constitute about $10 \%$ of J-stars (Lloyd Evans 1991), provide further evidence of a link with binarity (Lambert et al. 1990). Chemical pollution in masstransfer phases in binary systems during a nova-phase (Stehle $\&$ Ritter 1999) can result in peculiar surface abundances for the secondary star in a semi-detached state (Marks et al. 1997). Such pollution in nova systems presents a case of particular interest in the context of the observed ${ }^{13} \mathrm{C}$ enhancements in J-stars.

\subsection{Classical novae}

Novae are thermonuclear explosions in close binaries of the cataclysmic variable (CV) type (Warner 1995) with a WD accreting hydrogen-rich matter from a companion star undergoing Roche lobe overflow (RLOF). Among them, classical novae involve RLOF from a MS star to a WD (CO or ONe) in a close binary system resulting from a preceding common envelope (CE) phase (Paczynski 1976). At low accretion rates (below $10^{-7} M_{\odot} \mathrm{yr}^{-1}$ ), hydrogen is compressed to degenerate conditions until ignition occurs, which leads to a thermonuclear runaway (TNR). Explosive hydrogen burning synthesizes some short-lived $\beta$-unstable nuclei (e.g. $\left.{ }^{13} \mathrm{~N},{ }^{15} \mathrm{O}\right)$ which are transported by convection to the cooler outer envelope where isotopes like ${ }^{13} \mathrm{C}$ are produced from subsequent $\beta$-decays. These decays release sufficient energy which causes an explosive outburst in the outer shells of the WD accompanied by mass ejection with typical velocities $10^{2}-10^{3} \mathrm{~km} \mathrm{~s}^{-1}$ (Gehrz et al. 1998).
The energetics of the eruption, including the speed and mass of the ejecta, as well as the ejecta composition, are determined by the mass of the underlying $\mathrm{WD}, M_{\mathrm{WD}}$, and the mass accretion rate, $\dot{M}$. In classical novae, $\dot{M}$ typically ranges between $10^{-10}-10^{-8} M_{\odot} \mathrm{yr}^{-1}$ (Nomoto et al. 2007). For $\dot{M}=\frac{\mathrm{d} M_{\text {stable }}}{\mathrm{d} t} \sim$ $10^{-7} M_{\odot} \mathrm{yr}^{-1}$, the accreted material can burn steadily resulting in growth of the WD mass possibly up to the Chandrasekhar limit leading to a Type Ia supernova (Whelan \& Iben 1973; Wheeler 1992; Hachisu et al. 1996).

\subsection{Nova nucleosynthesis}

Observations of nova ejecta often show $\mathrm{C}, \mathrm{N}$ and $\mathrm{O}$ overabundances with respect to solar indicating that there is some mixing between the core and the accreted envelope (Gehrz et al. 1998). The mixing between the accreted envelope and the underlying WD is a necessary condition both to power the explosion and to interpret the extra-solar metallicities observed in nova ejecta (Hernanz et al. 2001). Even though the exact mechanism and the extent of this mixing remain unclear, the WD core composition plays a crucial role in the subsequent nucleosynthesis making it essential to distinguish between novae occurring on $\mathrm{CO}$ and ONe WDs (Glasner et al. 2012) .

\subsection{1. ${ }^{13} \mathrm{C}$ production in novae}

The synthesis of ${ }^{13} \mathrm{C}$ in novae is initiated through ${ }^{12} \mathrm{C}(\mathrm{p}, \gamma){ }^{13} \mathrm{~N}$ when the temperature at the base of the accreted material on the WD surface reaches about $10^{7} \mathrm{~K}$ as required for the cold $\mathrm{CNO}$ cycle to operate. The fate of ${ }^{13} \mathrm{C}$ is determined by the competition between destruction via ${ }^{13} \mathrm{C}(\mathrm{p}, \gamma){ }^{14} \mathrm{~N}$ which operates near the burning shell, and production by means of ${ }^{13} \mathrm{~N}\left(\beta^{+}\right){ }^{13} \mathrm{C}$ in the outer, cooler layers of the envelope where a fraction of ${ }^{13} \mathrm{~N}$ is carried to via convection just above the burning shell. The Jose \& Hernanz (1998) nova models show much higher overproduction of ${ }^{13} \mathrm{C}$ for $\mathrm{CO}$ novae as compared to ONe novae. This is expected primarily because of the higher initial ${ }^{12} \mathrm{C}$ content of COWDs, and the operation of the hot CNO cycle when ${ }^{13} \mathrm{~N}(\mathrm{p}, \gamma){ }^{14} \mathrm{O}$ is faster than ${ }^{13} \mathrm{~N}\left(\beta^{+}\right){ }^{13} \mathrm{C}$ (at base temperatures $>10^{8} \mathrm{~K}$ ) in more massive ONe WDs.

All of these model calculations assume a uniform WD composition without explicitly taking into account predictions from detailed evolutionary calculations. The nova ejecta composition depends critically on the initial composition of the WD material mixed with the accreted matter during explosion, and the predicted yields could well show larger enhancements of heavier (CNO) elements if outer layers of the underlying WD has a non-uniform composition (e.g. $\mathrm{C}: \mathrm{O}=50: 50$ for a $\mathrm{CO}$ nova). This could also account for the wide range of $\mathrm{C} / \mathrm{O}$ observed in nova ejecta (Gehrz et al. 1998). José et al. (2004) refer to a $0.6 M_{\odot} \mathrm{CO}$ WD model with initial C:O $=60: 40$ that gives ejecta with $\mathrm{C} / \mathrm{O}>1$. Such a case is of particular interest in context of carbon $\left({ }^{13} \mathrm{C}\right.$ in particular) pollution in nova explosions due to re-accretion of ejecta from the WD on its companion, and is considered in a systematic approach as detailed in the following section.

\section{Method}

This section outlines the nova re-accretion model we use to investigate pollution in MS companions to WDs (WD-companions hereafter) in nova binaries. The input physics and assumptions used in binary_c/nucsyn - our population nucleosynthesis code 
based on the BSE code of Hurley et al. (2002), extended to include various nucleosynthesis algorithms (Izzard et al. 2004, 2006, 2009) - are also described.

\subsection{Nova re-accretion model}

We use a simple geometric prescription to estimate the fraction of ejected mass re-accreted by the WD-companion (secondary) during the nova-outbursts following the approach used to study chemical pollution of the secondary during novae by Scott et al. (1994). Though nova shells are often observed to be asymmetric, this is most likely caused by the interaction of the expanding shells with the secondary star (Warner 1995). Hence, in order to estimate the amount of ejected material that is accreted back on the surface of the secondary, it is justifiable to assume a spherically symmetric outburst with a geometric factor for the fraction of the nova-ejecta intercepted by the secondary,

$f_{\text {nova }}=\frac{\pi R_{2}^{2}}{4 \pi a^{2}}$

which is the ratio of the cross-sectional area of the secondary star of radius $R_{2}$ to the area of a sphere of radius equal to the binary separation $a$. The mass of material re-accreted by the secondary star is thus given by,

$\Delta M_{\mathrm{re}-\mathrm{acc}}=\left(\frac{R_{2}}{2 a}\right)^{2} \Delta M_{\mathrm{ej}}$,

where $\Delta M_{\mathrm{ej}}$ is the mass ejected during the outburst. During the nova (semi-detached) phase when the secondary overfills its Roche lobe, $R_{2}$ is approximately the Roche lobe radius $R_{\mathrm{L}, 2}$, and hence Eq. (1) gives the fraction $f_{\text {nova }}$ as a analytic function of the mass ratio.

Equation (2) provides an upper estimate for the amount of material re-accreted, assuming all of the nova ejecta intercepted by the secondary sticks on to its surface. Detailed (3D) hydrodynamical studies also predict significant re-accretion of the ejecta in nova explosions on the surface of the WD companion (Campbell et al. 2010) and justify our simple choice for the prescription for re-accretion also used in previous works investigating similar effects of nova pollution (e.g. Marks et al. 1997).

\subsection{Nova input physics}

During novae, we assume that all but a small fraction $\epsilon=$ 0.001 of the accreted material is ejected in the explosion so that the WD does not grow significantly (Epelstain et al. 2007). Jose \& Hernanz (1998) yields are used for the composition of the ejecta of classical novae with WD masses in the range of $0.8-1.35 M_{\odot}$ including both CO \& ONe WD models, along with an additional $0.6 M_{\odot}$ CO-WD explosion model provided by Jose (priv. comm.).

All the CO-WD nova models assume a uniform composition $\mathrm{C}: \mathrm{O}=50: 50$ as initial input for the nucleosynthesis calculations in the explosion. However, a low mass CO-WD is expected to be carbon-rich $(\mathrm{C} / \mathrm{O}>1)$ in its outer layers that are mixed into the accreted envelope during the nova explosion (cf. Fig. 1). To account for this, an enhanced $\mathrm{C} / \mathrm{O} \sim 2$ is adopted for the ejecta composition of this $0.6 M_{\odot} \mathrm{CO}-\mathrm{WD}$ model. This choice is well within the observed range of ejecta compositions for classical novae as listed in the compilation of Gehrz et al. (1998), with even higher $\mathrm{C} / \mathrm{O}$ for the ejecta of CO-novae e.g. V842 Cen with a estimated $\mathrm{C} / \mathrm{O} \sim 5$ (Andrea et al. 1994).

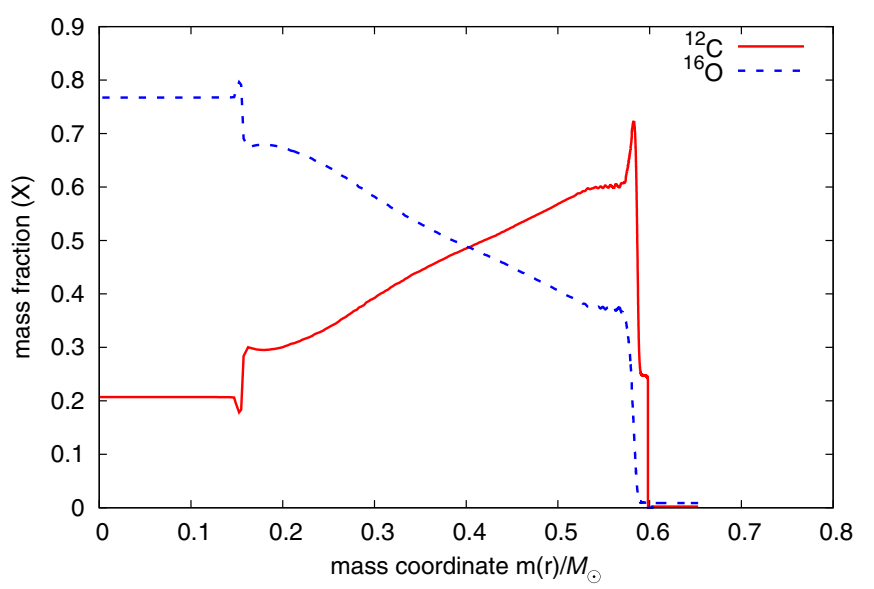

Fig. 1. Mass fractions of carbon and oxygen in the core of a TPAGB star with initial $M=3 M_{\odot}$ and $Z=0.02$ obtained using the MESA code (Paxton et al. 2013) illustrating that the outermost layers with mass coordinate $m(r) \gtrsim 0.5 M_{\odot}$ of a $0.6 M_{\odot} \mathrm{CO}-\mathrm{WD}$ are expected to have $\mathrm{C} / \mathrm{O}>1$ (by number).

\subsubsection{Common envelope prescription}

The evolution of all CV systems, including those that go through a nova phase, necessarily involves a CE phase which shrinks the orbital separation allowing a subsequent semi-detached phase of mass-transfer from the secondary (e.g. a MS star for classical novae). Hence, the CE phase plays an important role in determining which binary systems go through a nova phase after the envelope is ejected. We treat the CE phase using the $\alpha$-formalism of Paczynski (1976) assuming that the orbital energy release during the inspiral is transferred to the envelope with an efficiency $\alpha_{\mathrm{CE}}$, and can expand and eject at least some of the envelope (Hurley et al. 2002). The envelope binding energy is parametrized as,

$E_{\text {bind }}=-\frac{G M_{\mathrm{env}} M_{\mathrm{c}}}{\lambda R}$,

where $M_{\mathrm{env}}, M_{\mathrm{c}}$ and $R$ are the envelope mass, core mass and radius of the star and $\lambda$ is a measure of central condensation of the star that is calculated from detailed stellar models (Dewi \& Tauris 2000). The choice of the parameter $\alpha_{\mathrm{CE}}$ is critical for any population synthesis study involving nova binaries (Nelson et al. 2004). It is most probably not a constant (Davis et al. 2012) though population synthesis models usually assume a constant $\alpha_{\mathrm{CE}}<1$. Our systematic approach to constrain $\alpha_{\mathrm{CE}}$ for nova systems, as outlined in Appendix A, involves estimating the expected rate of novae in our Galaxy $(Z=0.02)$ in order to compare with existing observational constraints on the Galactic nova rate (Shafter 2002). Based on the results of our calculations (cf. Table A.1) we adopt a constant $\alpha_{\mathrm{CE}}=0.2$ for our population synthesis work which is also consistent with recent observational studies of post-CE binaries constraining $\alpha_{\mathrm{CE}}$ (Zorotovic et al. 2010; De Marco et al. 2011), and briefly discuss the systematic effect of this choice on our population synthesis predictions.

\section{3. binary_c/nucsyn}

To investigate the re-accretion of nova ejecta by WD-companions according to the prescription described in Sect. 2.1, the binary_c/nucsyn code is used to evolve grids of binaries at metallicities $Z=0.008$ and 0.02 for a range of initial primary $\left(M_{1}\right)$ and secondary $\left(M_{2}\right)$ masses with $M_{1}>M_{2}$, and initial separations $(a)$ which give nova systems within a Hubble 
time (13.7 Gyr). The following limits are therefore chosen for $M_{1}, M_{2}$ and $a$,

$M_{1}: 0.8-6 M_{\odot}$,

$M_{2}: 0.1 M_{\odot}-M_{1}$ and

$a: 10-10^{4} R_{\odot}$.

\subsubsection{Initial distributions}

The initial distributions of binary parameters $M_{1}, M_{2}$ and $a$ are given by the functions $\Psi\left(\ln M_{1}\right), \Phi\left(\ln M_{2}\right)$ and $\chi(\ln a)$ with,

$\Psi(\ln M)=M \xi(M)$,

where $\xi(M)$ is the initial mass function (IMF) of Kroupa et al. (1993). The distribution $\Phi$ for $M_{2}$ is chosen to be flat in the mass ratio $q=M_{2} / M_{1}$ and the separation distribution $\chi$ is taken to be flat in $\log a$, to facilitate comparison with previous works of Politano et al. (2010), Nelson et al. (2004).

\subsubsection{Binary fraction}

We assume a constant binary fraction $f_{\text {bin }}=0.5$ which corresponds to an equal number of single and binary systems in our stellar population (Duquennoy \& Mayor 1991).

\subsubsection{Binary grid}

With the above choices for initial binary parameters, a logarithmic grid is set up in $M_{1}-M_{2}-a$ space for all binary stars and in $M$ (initial mass with same choice of distribution and range as $M_{1}$ ) for single stars. The grid is split into $n$ stars per dimension such that each star represents the centre of a logarithmic grid-cell of size $\delta V$ where,

$\delta V=\left\{\begin{array}{cc}\delta \ln M & \text { single stars, } \\ \delta \ln M_{1} \delta \ln M_{2} \delta \ln a & \text { binary stars, }\end{array}\right.$

with

$\delta \ln x=\frac{\ln x_{\max }-\ln x_{\min }}{n-1}$,

where $x$ represents $M, M_{1}, M_{2}$ or $a$ and $x_{\min }$ and $x_{\max }$ are the grid limits. The probability for the $i$ th model in the grid is given by,

$p_{i}=\left\{\begin{array}{cr}\Psi\left(\ln M_{i}\right) \delta V & \text { single stars, } \\ \Psi\left(\ln M_{1 i}\right) \Phi\left(\ln M_{2 i}\right) \chi\left(\ln a_{i}\right) \delta V & \text { binary stars, }\end{array}\right.$

where

$\sum_{i} p_{i}=1$,

over the entire range of parameter space i.e. over masses (e.g. over $M$ for single stars) and separations. Hence for binaries,

$\sum_{i}=\sum_{M_{1}=0.8 \mathrm{M}_{\odot}}^{M_{1}=6 \mathrm{M}_{\odot}} \sum_{M_{2}=0.1}^{M_{1}} \sum_{\mathrm{M}_{\odot}}^{10^{4} \mathrm{R}_{\odot}}$

\subsubsection{Population synthesis}

The re-accretion model as outlined in Sect. 2.1 is applied to grids of binary models at a fixed metallicities $Z=0.02$ (solar) and 0.008 (LMC). The initial abundances are chosen to be solarscaled based on the values of Anders \& Grevesse (1989). The number of stars of a certain type (e.g. a C or J-star) is counted by calculating the probability of the existence of the stellar system (single or binary) given by Eq. (8) and the time it spends in the evolutionary phase of interest $\Delta t_{i}$ from,

$N=\sum_{i} S \times p_{i} \Delta t_{i} \delta \times\left\{\begin{array}{cc}\left(1-f_{\text {bin }}\right) & \text { single stars, } \\ f_{\text {bin }} & \text { binary stars }\end{array}\right.$

where $\delta=1$ if the star is in the phase of interest (and zero otherwise), the binary fraction $f_{\text {bin }}=0.5=1-f_{\text {bin }}$ and $S=7.086 \mathrm{yr}^{-1}$ is the (constant) star formation rate obtained following the prescription of Hurley et al. (2002). Thus, for C-stars, $\delta=1$ if $\mathrm{C} / \mathrm{O}>1$, and if in addition ${ }^{12} \mathrm{C} /{ }^{13} \mathrm{C}<10$, they also classify as J-stars. Also, $\mathrm{C}$ and J-stars are only counted as giants (stellar types GB, EAGB, TPAGB; Hurley et al. 2002) to compare with the statistics from observational surveys since luminosity estimates indicate that the stars are all giants. The number of J-type stars $\left(N_{\mathrm{J}}\right)$ is then calculated according to Eq. (11), along with the total number of C-stars $\left(N_{\mathrm{C}}\right)$ to predict the ratio $N_{\mathrm{J}} / N_{\mathrm{C}}$ giving the frequency of J-stars among C-stars.

\section{Results}

The evolution of nova binaries that are of interest in the reaccretion scenario for J-stars is presented in this section followed by an estimate for the number fraction of such nova-polluted C-stars expected to evolve as J-stars.

\subsection{Evolution of WD-companions in nova binaries}

The orbital evolution of binary systems that emerge from a first CE phase as a WD-MS binary depends on the mass ratio $q_{\text {donor }}$ (Pylyser \& Savonije 1988) given by,

$q_{\mathrm{donor}}=\frac{M_{\mathrm{donor}}}{M_{\mathrm{WD}}}$,

where $M_{\text {donor }}$ is the mass of the WD-companion. Assuming conservative mass-transfer, the orbital separation decreases during the nova phase if $q_{\text {donor }}>1$, and $M_{\text {donor }}$ decreases until $q_{\text {donor }}<1$. Thereafter, the orbit widens and the ultimate fate of such a system is a detached state with $M_{\text {donor }}$ being too low to evolve up to the giant branch within a Hubble time. Hence such systems are not interesting as progenitors for nova-polluted J-stars.

\subsubsection{Post-nova mergers as J-stars}

The ultimate fate of systems which keep $q_{\text {donor }}>1$ during the nova phase depends critically on the rate of mass-transfer, $\dot{M}$. The systems in Table 1 represent typical cases in which the binary eventually merges into a giant single star that satisfies the adopted criteria for J-stars (Sect. 2.3.4). Such post-nova mergers classify into two distinct binary evolutionary channels depending on the evolution of mass-transfer during the nova phase:

1. Coalescence $(C O A L)$ : if the nova phase continues with $\dot{M}<$ $10^{-7} M_{\odot} \mathrm{yr}^{-1}$ until the WD companion (donor) becomes a low-mass $\left(M_{\text {donor }} \simeq 0.7 M_{\odot}\right)$ MS star, mass-transfer to the 
Table 1. Initial binary parameters $M_{1}, M_{2}$ and $a$ for typical post-nova merger progenitors with for $Z=0.008, \alpha_{\mathrm{CE}}=0.2$.

\begin{tabular}{lccccccc}
\hline \hline$M_{1} / M_{\odot}$ & $M_{2} / M_{\odot}$ & $a / R_{\odot}$ & Case & $\mathrm{C} / \mathrm{O}$ & ${ }^{12} \mathrm{C} /{ }^{13} \mathrm{C}_{\mathrm{J}}$ & $M_{\mathrm{J}} / M_{\odot}$ & $M_{\mathrm{Bol}, \mathrm{J}}$ \\
\hline 1.63 & 1.20 & 363.1 & $\mathrm{COAL}-\mathrm{A}$ & 1.03 & 2.46 & 1.21 & -3.48 \\
2.15 & 0.78 & 631.0 & $\mathrm{COAL}-\mathrm{B}$ & 0.6 & 9.00 & 1.29 & -4.69 \\
1.71 & 0.94 & 631.0 & $\mathrm{CE}-\mathrm{A}$ & 1.17 & 2.09 & 1.02 & -2.25 \\
2.05 & 1.39 & 524.8 & $\mathrm{CE}-\mathrm{B}$ & 0.82 & 3.16 & 1.63 & -5.24 \\
\hline
\end{tabular}

Notes. The post-nova merger emerges with surface $\mathrm{C} / \mathrm{O}$ as given in Col. 5 . The J-suffix denotes the instant at which the merger has both $\mathrm{C} / \mathrm{O}>1$ and ${ }^{12} \mathrm{C} /{ }^{13} \mathrm{C}<10$ (J-star criteria) - thus $M_{\mathrm{J}}$ and $M_{\text {Bol, J }}$ respectively denote the mass and bolometric magnitudes (Eq. (13)) of the post-nova merger when it classifies as a J-star.

WD becomes dynamically unstable as the donor (MS) star becomes deeply convective and $q_{\text {donor }}(>1)$ also exceeds the critical mass-ratio $q_{\text {crit }}$ for stable RLOF (Hurley et al. 2002). All the material is accreted and swells up to form a giant envelope around the WD which becomes the core of a merged giant (TPAGB) star.

If re-accretion of nova-ejecta pollutes the WD-companion such that its surface $\mathrm{C} / \mathrm{O}>1$ and ${ }^{12} \mathrm{C} /{ }^{13} \mathrm{C}<10$, the coalesced (AGB) star classifies as a J-star. Such a typical system is labeled as COAL-A in Table 1 in which the merged star is a $1.2 M_{\odot}$ TPAGB star with a CO core mass $\sim 0.6 M_{\odot}$ and hence has a lower luminosity $L \sim 10^{3} L_{\odot}\left(\Rightarrow M_{\mathrm{Bol}}>-4\right)$ compared to typical AGB C(N)-stars with $L \sim 10^{4} L_{\odot}\left(\Rightarrow M_{\mathrm{Bol}}<-4\right)$ and $M \gtrsim 2 M_{\odot}$. However, following the nova-phase, if the WD-companion has surface $\mathrm{C} / \mathrm{O}<1$ but ${ }^{12} \mathrm{C} /{ }^{13} \mathrm{C}<10$, the merger can subsequently evolve on the $\mathrm{AGB}$ with $\mathrm{C} / \mathrm{O}>1$ because of TDU until its ${ }^{12} \mathrm{C} /{ }^{13} \mathrm{C}>10$, and classify as a J-star. A typical example is labeled as COAL-B in Table 1 with a higher luminosity $\left(M_{\mathrm{Bol}}<-4\right)$ as compared to the COAL-A system depending on their evolutionary phases on the AGB when they classify as J-stars. Figure 2 illustrates the differences in evolution of these two typical COAL systems through the nova-phase followed by the AGB phase of the nova-polluted merger that evolves into a J-star.

2. Common envelope $(C E)$ : for suitable initial parameters $\left(M_{1}\right.$, $\left.M_{2}, a\right)$, e.g. systems CE-A/B of Table 1, RLOF proceeds at the thermal rate of the WD-companion when it evolves as a Hertzsprung Gap (HG) star with a radiative or thin convective envelope. When the thermal rate is less than $10^{-7} M_{\odot} \mathrm{yr}^{-1}$, the nova phase continues until the WD-companion becomes a giant (GB) when mass-transfer is dynamically unstable and leads to a (second) CE phase. Consequently the system merges and the CO-WD becomes the core surrounded by a He-shell (core of the giant donor) resembling an EAGB star whose surface abundances are determined primarily by the donor star which suffers from nova-pollution. A typical case is labelled as CE-A in Table 1 with the merged EAGB star having both $\mathrm{C} / \mathrm{O}>1$ and ${ }^{12} \mathrm{C} /{ }^{13} \mathrm{C}<10$.

If the thermal rate exceeds $10^{-7} M_{\odot} \mathrm{yr}^{-1}$ during the RLOFphase, steady H-burning on the surface of the accreting WD ends the nova phase. Thereafter, the orbit continues to shrink leading to runaway mass transfer until the material accreted onto the WD swells up to form a giant star and the system also merges in a (second) CE-phase. Such an example is the CE-B case of Table 1 for which the CE merger outcome ascends the AGB with ${ }^{12} \mathrm{C} /{ }^{13} \mathrm{C}<10$ owing to previous nova-pollution until it becomes a $\mathrm{C}$-star when $\mathrm{C} / \mathrm{O}>1$ because of TDU. Thus it classifies as a J-star only for a brief phase $\left(\sim 10^{5} \mathrm{yr}\right)$ of its TPAGB until ${ }^{12} \mathrm{C} /{ }^{13} \mathrm{C}>10$ because ${ }^{12} \mathrm{C}$ is brought to the surface by TDU. The evolution of this system is shown in Fig. 3 for the nova phase preceding the $\mathrm{CE}$, and the AGB phase of the merged star that becomes a J-star on the EAGB for the CE-A system but requires TDU on the TPAGB for the CE-B system.

All the above examples of nova-binaries considering pollution of the WD-companion stars present interesting outcomes that can classify as J-stars with their evolutionary status determined by the preceding nova-phase of the progenitor systems. Accordingly, they vary in their properties (viz. masses, luminosities, chemistry) depending on whether the WD-companion re-accretes enough nova ejecta to evolve with $\mathrm{C} / \mathrm{O}>1$, along with ${ }^{12} \mathrm{C} /{ }^{13} \mathrm{C}<10$.

Thus, the nova re-accretion scenario predicts mergers that evolve as J-stars with very low ${ }^{12} \mathrm{C} /{ }^{13} \mathrm{C}(<4)$ ratios and luminosities $L \sim 10^{3} L_{\odot}\left(\Rightarrow M_{\mathrm{Bol}}>-4\right)$ unlike typical AGB C-stars undergoing $\mathrm{HBB}$ with low ${ }^{12} \mathrm{C} /{ }^{13} \mathrm{C}$. Post-nova merger systems like COAL-A and CE-A are C-rich from the re-accretion of nova-ejecta and thus classify as J-stars even without TDU. On the other hand, cases COAL-B and CE-B which emerge as postnova mergers necessarily require TDU to have $\mathrm{C} / \mathrm{O}>1$ and thus behave as more luminous J-stars on the TPAGB.

\subsubsection{Initial binary parameters for post-nova mergers}

Figure 4 shows the range of initial binary parameters ( $M_{1}$ vs. $M_{2}, M_{1}$ vs. $a$ ) for the post-nova merger s that evolve to become J-stars. The COAL mergers that classify as J-stars mostly originate from binary systems with WD masses close to $0.6 M_{\odot}$ for $M_{1}<3 M_{\odot}$. CE mergers mostly originate from systems with $M_{2}>1 M_{\odot}$ which involve thermal-timescale masstransfer (mostly on the HG) during RLOF.

\subsection{J-stars from Case-B RLOF mergers}

Unlike nova progenitor systems that emerge as a WD-MS binary following a CE phase, depending on their initial separation binaries also merge in the $\mathrm{CE}$ phase because of unstable mass transfer from a primary star on the GB to a MS secondary (Case-B RLOF). This is illustrated in Fig. 5 which distinguishes the evolutionary channel for these systems from the post-nova mergers (COAL/CE) as described in Sect. 3.1.1. The merged (GB) star has a lower helium core mass than expected for a single star of the same total mass at the beginning of the GB. The typical evolution of such an object on the TPAGB is shown in Fig. B.1 (cf. Appendix B), along with a single star model of similar total mass, which illustrates the difference in evolutionary time-scales for the two cases. As a result, they contribute significantly to the number of stars that classify as C and J-stars in our population synthesis calculations as described next. 


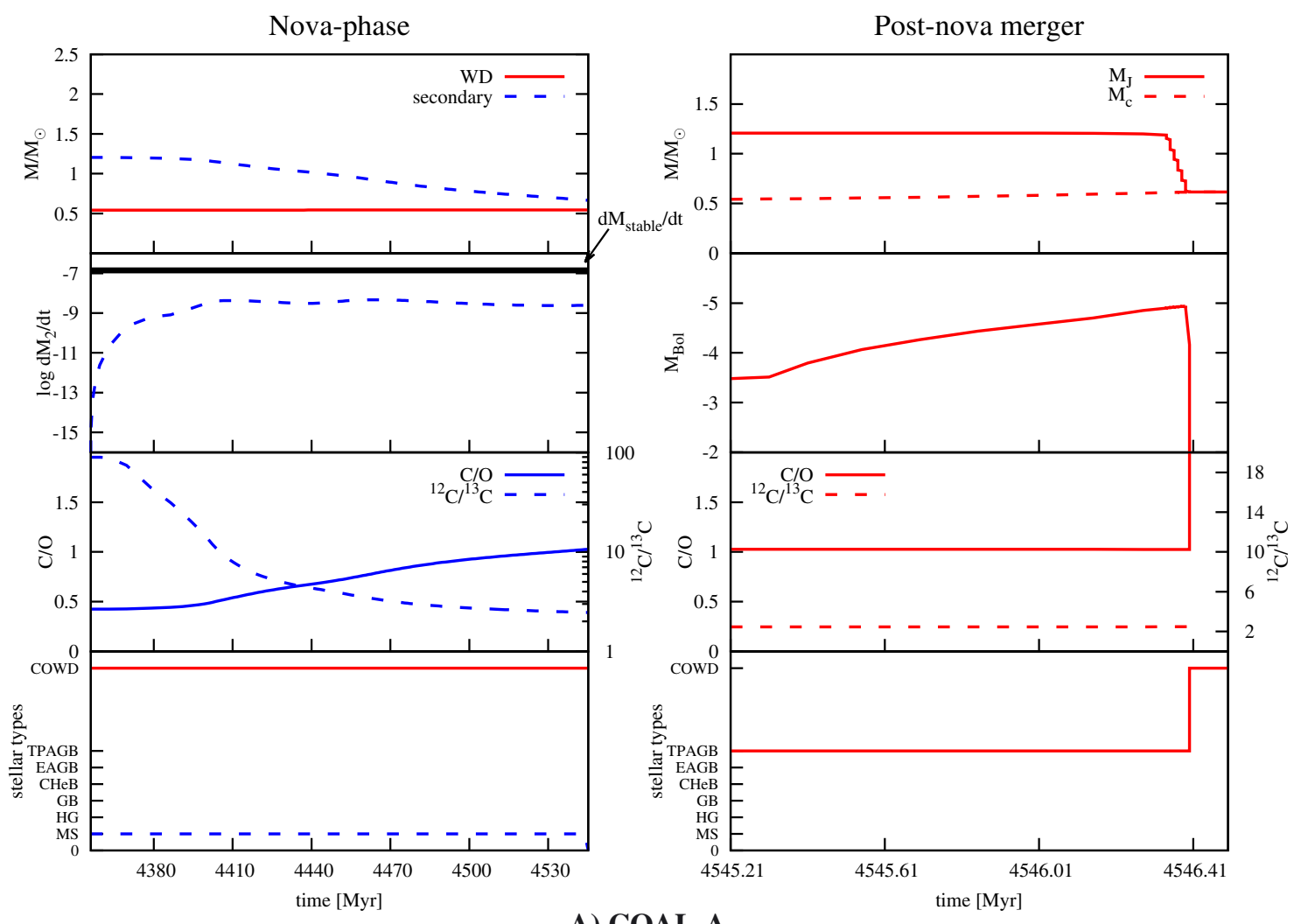

A) COAL-A

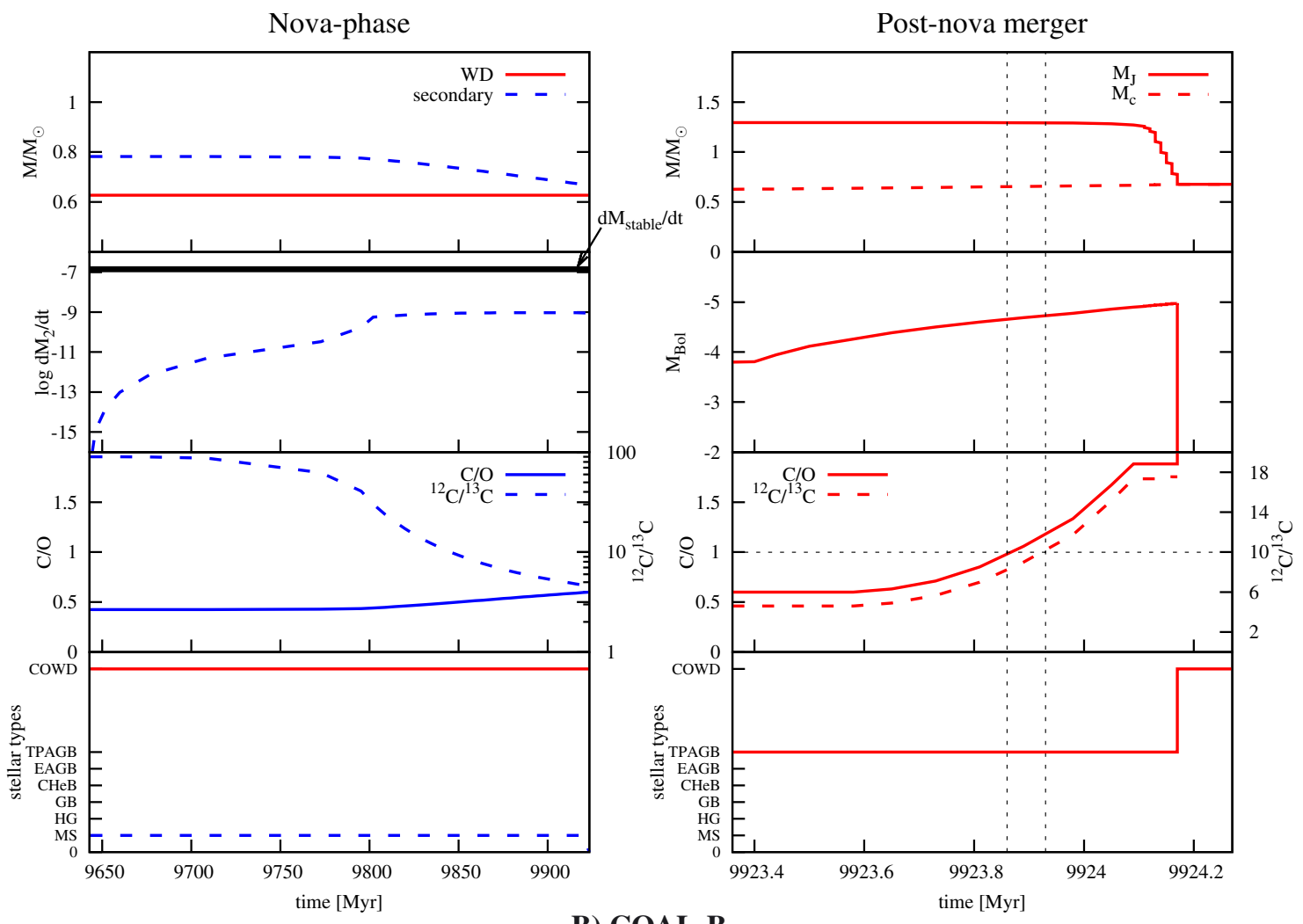

B) COAL-B

Fig. 2. Evolution of COAL type systems of Table 1. For each system i.e. COAL-A (Fig. A) and COAL-B (Fig. B), the top and bottom panels on the left show masses and evolutionary stages of both the primary (WD) and the secondary stars during the nova phase when the mass-transfer rate $\left(\frac{\mathrm{d} \dot{M}_{2}}{\mathrm{~d} t}\right)$ and surface chemistry $\left(\mathrm{C} / \mathrm{O},{ }^{12} \mathrm{C} /{ }^{13} \mathrm{C}\right)$ for the donor (secondary) star evolve with time as depicted in the second and third panels. The right hand panels follow the post-nova merger properties i.e. mass, core-mass, luminosity, surface chemistry and its stage of evolution (EAGB/TPAGB). The COAL-A merger classifies as a J-star for the entire duration of its AGB lifetime, as compared to the the COAL-B merger that has C/O > 1 during TDU on the TPAGB resulting in a much shorter J-star phase marked by the vertical dotted lines. 
S. Sengupta et al.: A nova re-accretion model for J-type carbon stars
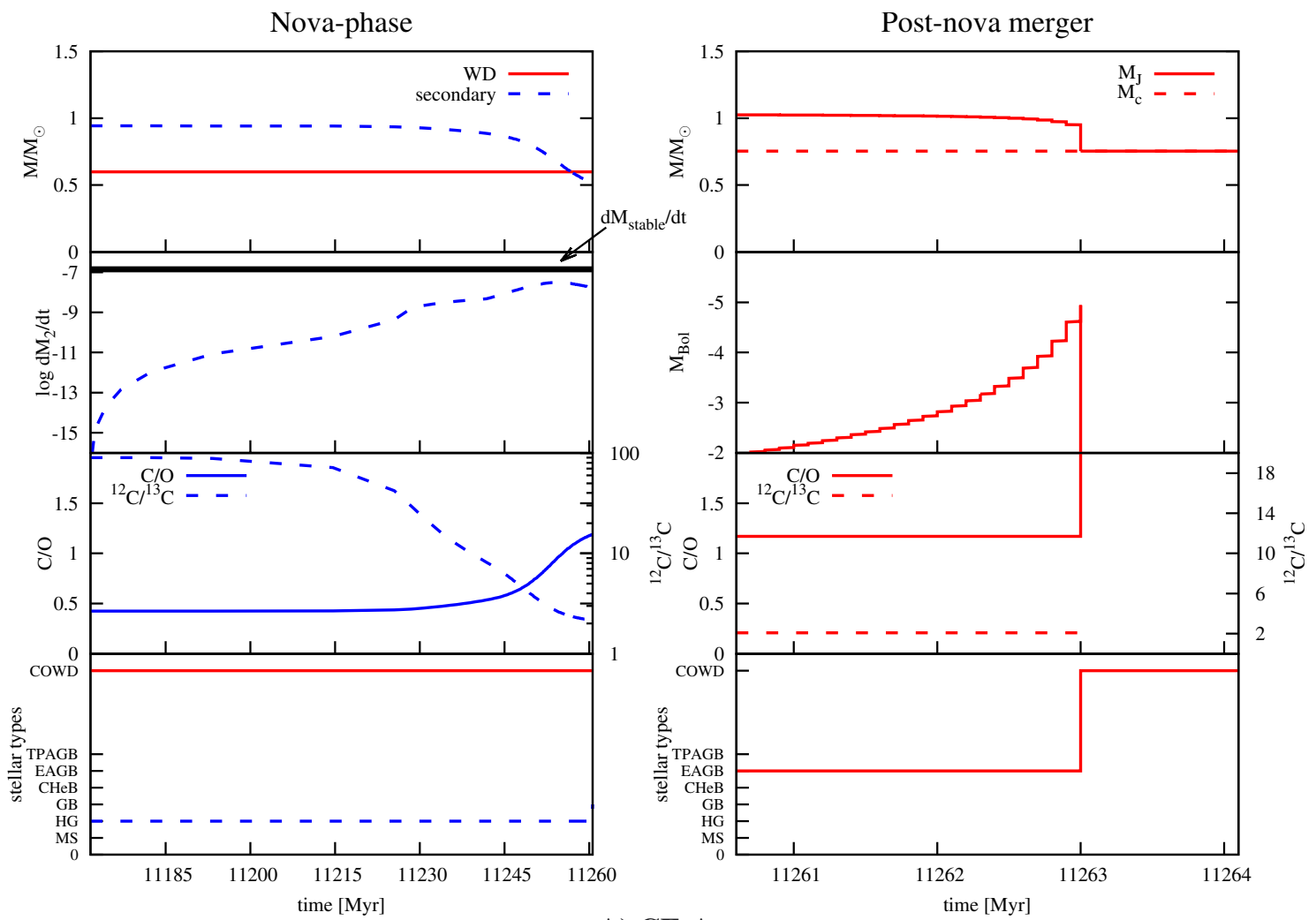

A) CE-A
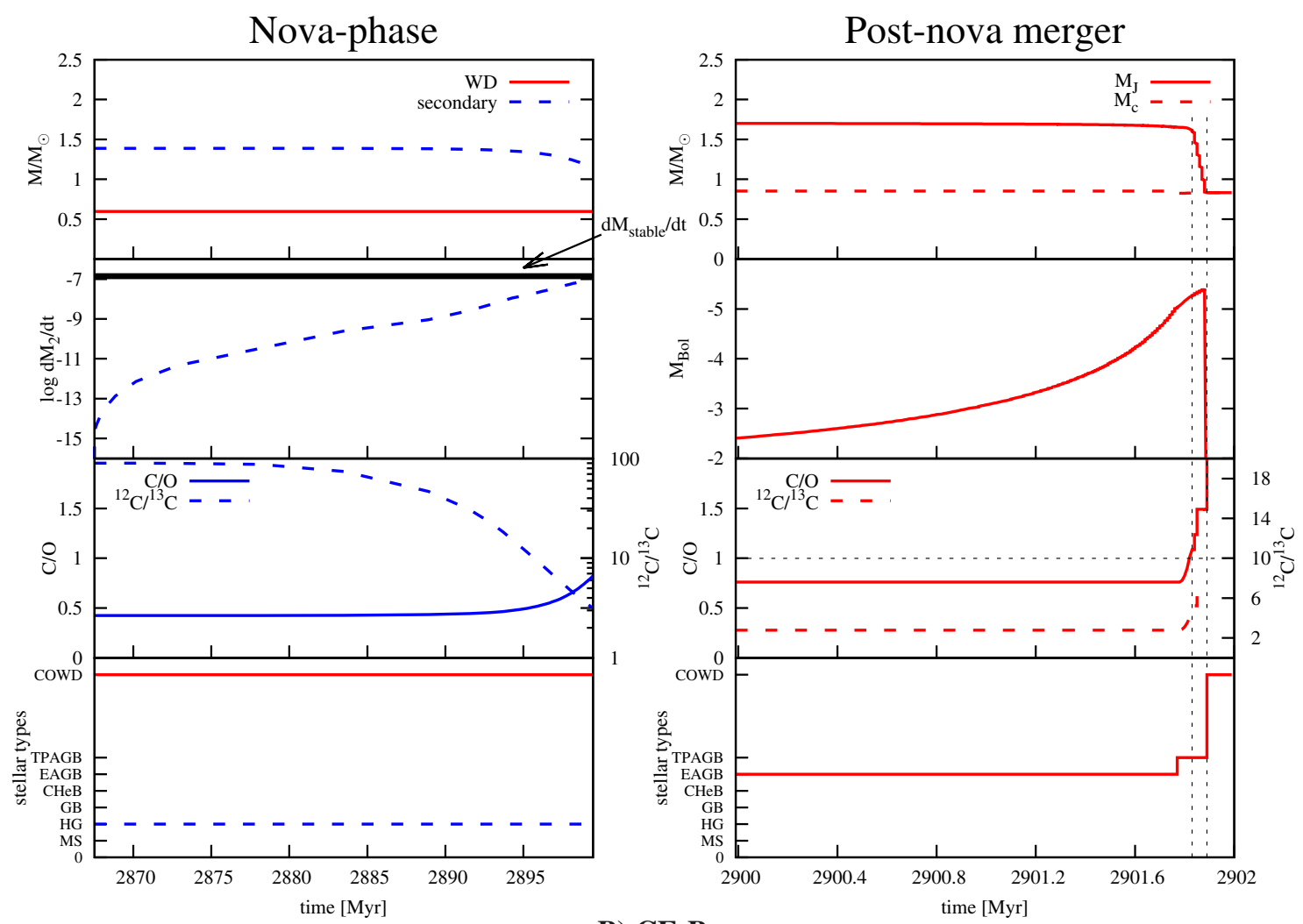

B) CE-B

Fig. 3. Evolution of CE type systems of Table 1. Refer to Fig. 2 caption for description of evolutionary stages shown. The CE-A merger exhibits properties of a J-star on the EAGB unlike the COAL-B merger which has C/O > 1 only during TDU and hence has a short phase on the TPAGB (marked by the dotted lines) when it classifies as a J-star. 

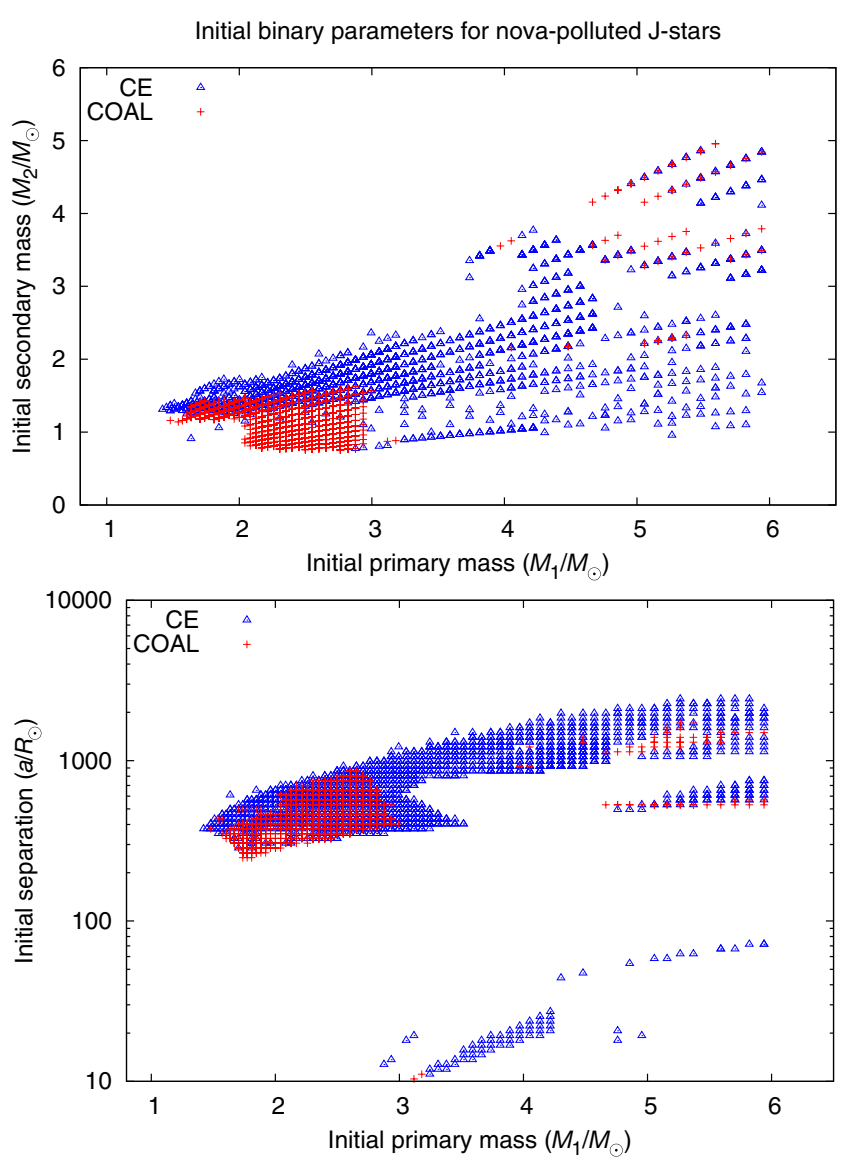

Fig. 4. Range of initial primary mass $M_{1}$, initial secondary mass $M_{2}$ and initial separation $a$ for nova-merger cases (COAL/CE) that form J-stars.

\subsection{Population synthesis}

With the method outlined in Sect. 2.3.4, a binary grid of resolution $100 \times 50 \times 100$ in $M_{1}-M_{2}-a$ space is evolved for $Z=0.008$ and $0.02, \alpha_{\mathrm{CE}}=0.2$ and $f_{\text {bin }}=0.5$ to estimate the total number of J-stars expected from the instances of nova-pollution as described in Sect. 3.1.1, as well as from HBB in both single and binary AGB stars.

\subsubsection{Observational selection effects}

Our population synthesis is performed considering possible selection effects in observations from existing surveys for J-stars. For instance in the LMC survey of Morgan et al. (2003), the $J, H, K$ magnitudes for all J-stars lead to bolometric magnitudes $M_{\mathrm{Bol}}<-2.8$ derived using the relation (as adopted in Hatzidimitriou et al. 2003),

$M_{\mathrm{Bol}}=K_{0}-d m+0.69+2.65(J-K)_{0}-0.67(J-K)_{0}^{2}$,

with a distance modulus $d m=18.45$ for the LMC (Westerlund 1997), a $K$-band absorption $A_{K}=0.02$ (Wood et al. 1983) along with mean reddening $E(J-K)=0.07$ (Costa \& Frogel 1996) to find the de-redenned colours denoted by $K_{0}=K-A_{K}$ and $(J-K)_{0}=(J-K)-E(J-K)$. We use this as a low-luminosity cut-off in our estimates for the number of $\mathrm{C}\left(N_{\mathrm{C}}\right)$ and $\mathrm{J}\left(N_{\mathrm{J}}\right)$ stars.

\subsection{2. $N_{\mathrm{J}} / N_{\mathrm{C}}$ ratio}

The number of stars of interest (i.e. $N_{\mathrm{J}}, N_{\mathrm{C}}$ ) is calculated as described in Sect. 2.3.4 for all systems both in our single and binary

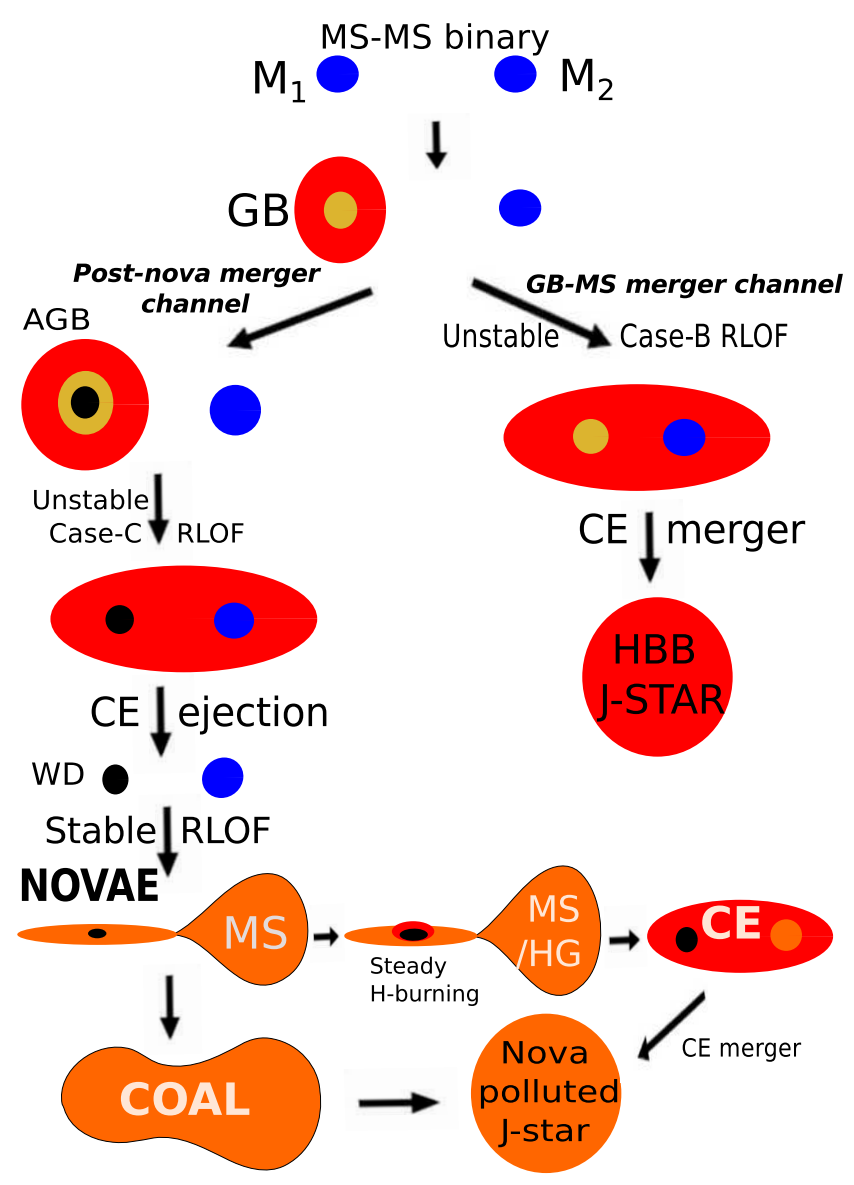

Fig. 5. Binary evolutionary channels which lead to mergers with properties of J-stars from re-accretion of nova-ejecta followed by COAL and CE mergers or HBB in AGB-phases of GB-MS (Case-B RLOF) mergers. The different stellar evolutionary phases are marked (with colours) as - blue: MS star, red: giant-envelope/CE, golden: He-core/WD, black: CO-core/WD, orange: nova-polluted star (MS/HG).

grids for metallicities $Z=0.008$ (LMC) and 0.02 (solar). The resulting $N_{\mathrm{J}} / N_{\mathrm{C}}$ ratio are presented in Table 2 , with the binary J-stars separated (in columns) according to their evolutionary origin i.e. post-nova merger or HBB. Furthermore, the difference in the values of the ratios between the upper and lower rows in each of these two columns occurs from counting Case-B RLOF mergers (as described in Sect. 3.2) which have considerably longer HBB-AGB phases compared to single AGB stars of same total mass (cf. Appendix B) and classify as J-stars. However, the total $N_{\mathrm{J}} / N_{\mathrm{C}}$ ratio considering all single $(\mathrm{HBB})$ and binary (merger) $\mathrm{J}$-stars is still below the observed fraction (10-15\%) for both $Z=0.008$ and 0.02 .

\subsubsection{Luminosity function for LMC J-stars}

The J-star luminosity function (JSLF) is constructed with the $N_{\mathrm{J}} / N_{\mathrm{C}}$ ratio binned in $M_{\mathrm{Bol}}$ intervals of $0.5 \mathrm{mag}$ (Fig. 6). In order to compare with observations, Eq. (13) is used to derive the bolometric magnitudes of all the 156 LMC J-stars of Morgan et al. (2003).

Figure 6 shows that the post-nova merger channel produces J-stars that are dimmer (with $M_{\mathrm{Bol}}>-5$ ) but much rarer compared to the HBB stars (with $M_{\mathrm{Bol}}<-6$ ). In contrast, the distribution constructed from the observed sample of Morgan et al. (2003) has the majority of J-stars dimmer than $M_{\mathrm{Bol}}=-6$. However, most of the J-stars with $M_{\text {Bol }}<-6$ result from Case-B 
Table 2. $N_{\mathrm{J}} / N_{\mathrm{C}}$ ratios for single (Col I) and binary (Col II) stars.

\begin{tabular}{|c|c|c|c|c|c|}
\hline \multirow{2}{*}{$Z$} & \multirow{2}{*}{$N_{\mathrm{J}} / N_{\mathrm{C}}(\%)$ from single $(\mathrm{HBB})$ stars $(\mathrm{I})$} & \multicolumn{2}{|c|}{$N_{\mathrm{J}} / N_{\mathrm{C}}(\%)$ from binaries (II) } & \multicolumn{2}{|c|}{$N_{\mathrm{J}} / N_{\mathrm{C}}(\%)(\mathrm{III})$} \\
\hline & & Post-nova mergers (II a) & HBB (II b) & Model (III a) & Obs. (III b) \\
\hline \multirow{2}{*}{0.008} & \multirow{2}{*}{0.71} & 3.0 & 0.27 & 1.95 & \multirow{2}{*}{10} \\
\hline & & $1.07^{*}$ & $4.75^{*}$ & 4.45 & \\
\hline \multirow{2}{*}{0.02} & \multirow{2}{*}{0.00} & 0.3 & 0.06 & 0.36 & \multirow{2}{*}{15} \\
\hline & & $0.01^{*}$ & $3.43^{*}$ & 3.44 & \\
\hline
\end{tabular}

Notes. The ratios in IIa and IIb marked by $*$ are calculated by including GB-MS (Case-B RLOF) mergers which have a longer HBB-phase compared to single AGB stars of similar total mass, and hence increase the $N_{\mathrm{C}}$ and $N_{\mathrm{J}}$ for binaries from HBB stars. The model (III a) $N_{\mathrm{J}} / N_{\mathrm{C}}$ is calculated by adding the contributions to $N_{\mathrm{J}}$ and $N_{\mathrm{C}}$ from both single (I) and binary stars (II a \& b), assuming a constant binary fraction of 0.5 . Observed statistics (III b) are from Morgan et al. (2003) and Abia \& Isern (2000) for the LMC and our Galaxy respectively.

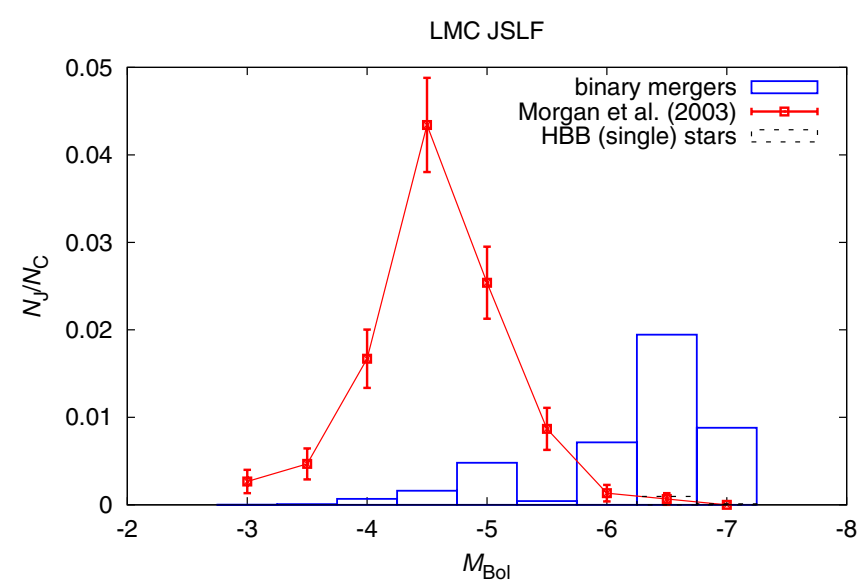

Fig. 6. Luminosity function of LMC J-stars (JSLF). The histograms binned in $M_{\mathrm{Bol}}$ intervals of $0.5 \mathrm{mag}$ show expected number fraction $\left(N_{\mathrm{J}} / N_{C}\right)$ of $\mathrm{J}$-stars among C-stars for HBB (single) stars and binary mergers (post-nova or Case-B RLOF) - compared with the JSLF constructed for the LMC J-stars of Morgan et al. (2003) with Poisson $\sqrt{n}$ error bars where $n$ is the number of stars in a particular $M_{\text {Bol }}$ bin.

RLOF whose post-merger evolution on the (TP)AGB is different compared to single (AGB) stars of similar masses (cf. Fig. B.1). The JSLF for the post-nova mergers is shown separately in Fig. 7 along with that of single and binary HBB-AGB stars excluding the Case-B RLOF mergers. It has closer resemblance to the JSLF of the stars from Morgan et al. (2003), with a higher fraction of low-luminosity J-stars as observed in the Morgan et al. (2003) survey, though the predicted $N_{\mathrm{J}} / N_{\mathrm{C}}$ is still lower and well below the observed fraction for the dimmer $\left(M_{\mathrm{Bol}}>-6\right) \mathrm{J}$-stars in the LMC.

\subsubsection{Number distributions}

The number distributions for masses $(M)$ and ${ }^{12} \mathrm{C} /{ }^{13} \mathrm{C}$ ratios of LMC $(Z=0.008) \mathrm{J}$-stars are shown in Fig. 8, excluding the HBB Case-B RLOF mergers (as described in Sect. 3.2). The postnova mergers have ${ }^{12} \mathrm{C} /{ }^{13} \mathrm{C}$ ratios lower than 4 , and low masses $\left(<2 M_{\odot}\right)$ unlike the HBB stars, since majority of the post-nova mergers owe their $\mathrm{C}$-enhancement to the re-accretion of $\mathrm{C}$-rich nova ejecta from a low-mass $\left(\sim 0.6 M_{\odot}\right) \mathrm{CO}-\mathrm{WD}$ and can evolve as J-stars without TDU on the AGB. The ${ }^{12} \mathrm{C} /{ }^{13} \mathrm{C}$ ratios remain low on the AGB for such systems e.g. COAL-A (cf. Table 1) whereas for HBB-stars $\left(>2 M_{\odot}\right)$ the ${ }^{12} \mathrm{C} /{ }^{13} \mathrm{C}$ ratios increase during TDU when they become $\mathrm{C}(\mathrm{J})$-stars.

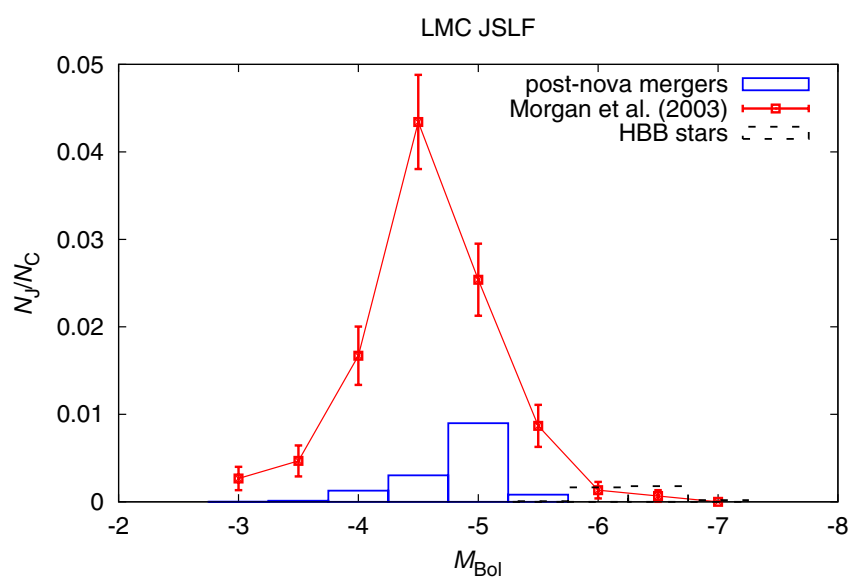

Fig. 7. Luminosity function of LMC J-stars (JSLF). Number fraction $\left(N_{\mathrm{J}} / N_{C}\right)$ of J-stars among C-stars for post-nova mergers and HBB-stars excluding Case-B RLOF binary mergers is shown with observed J-stars from the sample of Morgan et al. (2003) binned in $M_{\text {Bol }}$ intervals of $0.5 \mathrm{mag}$. The total $N_{\mathrm{J}} / N_{C}$ ratio is $\sim 2 \%$ for the nova-polluted mergers lower than the total $N_{\mathrm{J}} / N_{C}$ in Fig. $6(\sim 5 \%)$ and the observed fraction $(\sim 10 \%)$ of the stars in the sample of Morgan et al. (2003).

\section{Discussion}

The binary scenario for the origin of J-stars explored in this work illustrates the need for detailed evolutionary studies in several aspects of both single and binary star evolution. The nucleosynthesis associated both with nova explosions for low-mass CO-WDs as well the treatment of HBB during AGB evolution, particularly in context of binary mergers, require detailed models that account for the evolutionary history of the associated binary system. Particularly, detailed evolutionary calculations of RG-MS (Case-B RLOF) mergers are required to determine their evolutionary time-scales and nucleosynthesis (especially the phase with $\mathrm{HBB}$ ) on the AGB.

In the context of post-nova mergers that can exhibit properties associated with J-stars, other chemical peculiarities e.g. the $\mathrm{O}$-isotopic ratios, $\mathrm{N}$-enhancements, Li-enrichment, s-process abundances etc., need to be investigated and compared with observations (e.g. Hedrosa et al. 2013). To quantitatively predict these isotopic ratios for the J-stars expected from the novapollution scenario, a detailed low-mass CO-WD nova model is required providing ejecta composition for initial $\mathrm{C}: \mathrm{O}$ ratio different from a uniform one (50:50) as used in existing models of CO-WD explosions. Also, chemical pollution of the secondary (donor) star during the nova phase with carbon rich ejecta should influence the subsequent nova explosions when RLOF 

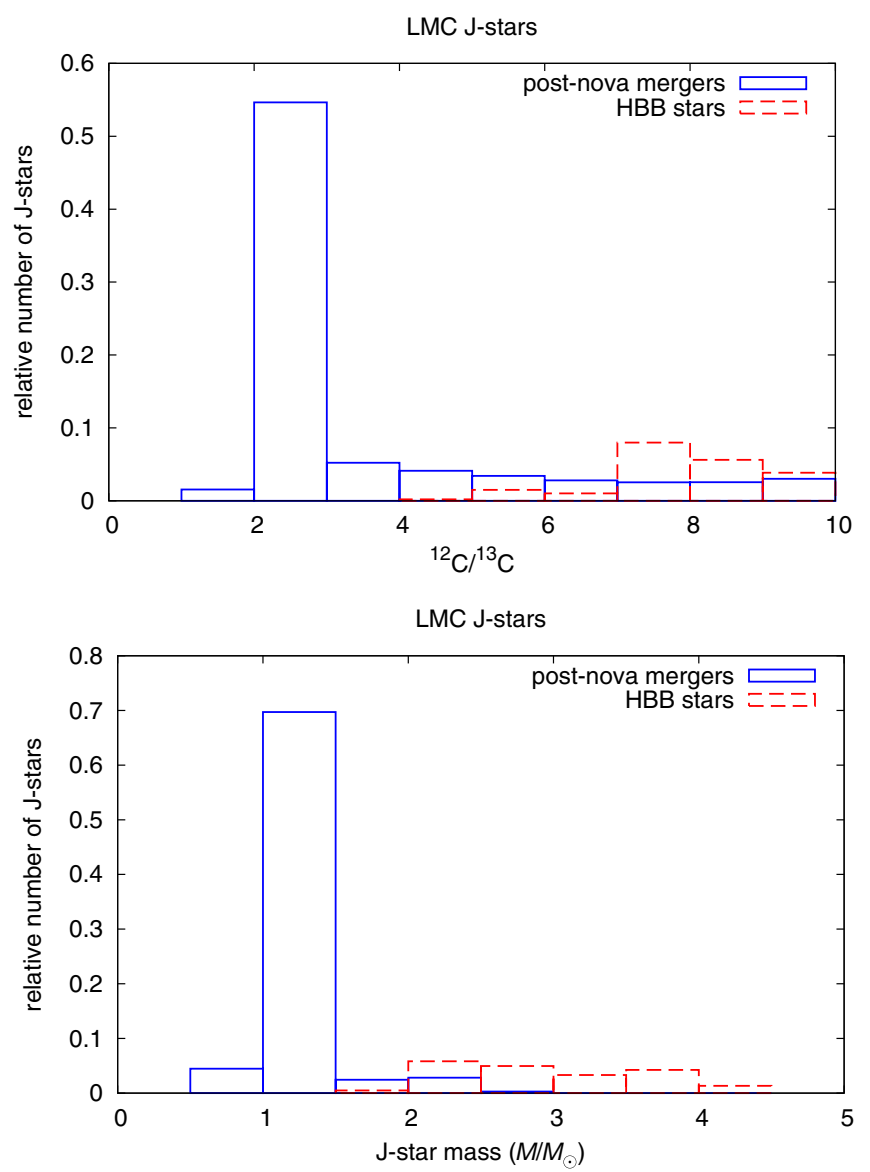

Fig. 8. Distributions of ${ }^{12} \mathrm{C} /{ }^{13} \mathrm{C}$ (upper panel) and masses (lower panel) for J-stars including post-nova mergers and HBB-stars but excluding Case-B RLOF binary mergers for $Z=0.008$. The relative number of $\mathrm{J}$-stars is the ratio of number of J-stars in each bin to the total number of J-stars.

from the nova-polluted donor transfers some of the material from its surface back to the WD. However, investigating such an effect requires nova models with varying accretion compositions which is beyond the scope of the present study.

The systematic uncertainties associated with our prediction for the $N_{\mathrm{J}} / N_{\mathrm{C}}$ ratio for the post-nova merger channel (without HBB stars) include our choice of the CE ejection efficiency parameter $\alpha_{\mathrm{CE}}$. The post-CE separations for the WD-MS nova progenitor systems increase with $\alpha_{\mathrm{CE}}$ which prevents RLOF from the WD-companion during its MS/HG phase of evolution and a subsequent nova phase for the binary. We adopt a constant $\alpha_{\mathrm{CE}}=0.2$ based on our estimate for the Galactic nova rate (refer Appendix A) which is constrained by observations (Shafter 2002). With regard to other assumptions in our modelling of nova systems, the use of a nearly constant WD mass in the nova phase is justifiable within the usual range of classical novae mass transfer rates $\left(\dot{M} \sim 10^{-10}-10^{-8} M_{\odot} \mathrm{yr}^{-1}\right)$ based on detailed models which investigate the ratio of mass ejected to that accreted in outbursts for the entire range of $M_{\mathrm{WD}}$ (Yaron et al. 2005). We also use a constant binary fraction $f_{\text {bin }}=0.5$ in our population synthesis since the post-nova mergers that classify as J-stars evolve from low-mass WD-MS systems and in view of existing observations estimating the binary fraction for this range ( 1 $M_{\odot}$ ) of stellar masses (Duchêne \& Kraus 2013).

\section{Conclusions}

This work investigates the effect of chemical pollution of WD companions in nova binaries because of re-accretion of nova ejecta and their subsequent evolution with observed properties of J-stars. The re-accretion model (Sect. 2.1) is applied to a population of binary systems using the population nucleosynthesis code binary_c/nucsyn to predict the properties of post-nova systems that evolve as giant stars having both $\mathrm{C} / \mathrm{O}>1$ and ${ }^{12} \mathrm{C} /{ }^{13} \mathrm{C}<10$ - properties characteristic to J-stars. We identify systems which merge (COAL or CE) as a result of unstable (dynamical or thermal) mass-transfer following a nova phase during which the material ejected from the WD pollutes its companion with enough carbon (including ${ }^{13} \mathrm{C}$ ) to have ${ }^{12} \mathrm{C} /{ }^{13} \mathrm{C}<10$.

The post-nova mergers evolve as AGB J-stars with a lowmass WD as its core and the companion absorbed into its envelope. Such low mass $\left(<2 M_{\odot}\right)$ AGB stars have low ${ }^{12} \mathrm{C} /{ }^{13} \mathrm{C}$ and are dimmer than $M_{\mathrm{Bol}}=-5$, resembling observed J-stars in the LMC that cannot otherwise be explained with single star (HBB) models. However, our population synthesis study shows that this channel together with HBB (single or binary) stars cannot account for the observed fraction of J-stars among C-stars in the LMC and our Galaxy. It indicates that other channels for the origin of J-stars need to be taken into account in future studies aiming to explain the observed frequency of J-stars. The GB-MS (Case B RLOF) mergers that possibly behave differently from normal (single) AGB stars are of particular interest and detailed evolutionary calculations are necessary to determine their evolutionary time-scales. Other binary scenarios such as HeWD-giant mergers (Zhang \& Jeffery 2013) should also be investigated in a population synthesis framework to explain the observed fraction of J-stars among C-stars. Such scenarios could also lead to possible evolutionary connections with other classes of C-stars e.g. CEMP-no stars which show chemical peculiarities common to J-stars at much lower metallicities associated with the Galactic halo.

Acknowledgements. We acknowledge the anonymous referee for useful comments and interesting suggestions for further studies. SS thanks K. Ohnaka for sharing his observational expertise throughout the course of this work and for his valuable comments to improve the manuscript. Special thanks also go to J. Jose and R.J. Stancliffe for providing their models for this work. S.S. received the financial support from the Bonn-Cologne Graduate School for Physics and Astronomy and the Bonn International Graduate School and is a member of the International Max Planck Research School for Astronomy and Astrophysics at the Universities of Bonn and Cologne. R.G.I. and H.H.B.L. thank the Alexander von Humboldt foundation for funding their positions in Bonn.

\section{References}

Abia, C., \& Isern, J. 2000, ApJ, 536, 438

Anders, E., \& Grevesse, N. 1989, Geochim. Cosmochim. Acta, 53, 197

Andrea, J., Drechsel, H., \& Starrfield, S. 1994, A\&A, 291, 869

Boothroyd, A. I., Sackmann, I.-J., \& Wasserburg, G. J. 1995, ApJ, 442, L21

Bouigue, R. 1954, Annales d'Astrophysique, 17, 104

Campbell, S., Jose, J., Cabezon, R., \& García-Berro, E. 2010, in 11th Symposium on Nuclei in the Cosmos, PoS(NIC XI)203

Costa, E., \& Frogel, J. A. 1996, AJ, 112, 2607

Davis, P. J., Kolb, U., \& Knigge, C. 2012, MNRAS, 419, 287

De Marco, O., Passy, J.-C., Moe, M., et al. 2011, MNRAS, 411, 2277

Dewi, J. D. M., \& Tauris, T. M. 2000, A\&A, 360, 1043

Duchêne, G., \& Kraus, A. 2013, ARA\&A, 51, 269

Duquennoy, A., \& Mayor, M. 1991, A\&A, 248, 485

Epelstain, N., Yaron, O., Kovetz, A., \& Prialnik, D. 2007, MNRAS, 374, 1449

Gehrz, R. D., Truran, J. W., Williams, R. E., \& Starrfield, S. 1998, PASP, 110, 3

Glasner, S. A., Livne, E., \& Truran, J. W. 2012, MNRAS, 427, 2411

Hachisu, I., Kato, M., \& Nomoto, K. 1996, ApJ, 470, L97

Hatzidimitriou, D., Morgan, D. H., Cannon, R. D., \& Croke, B. F. W. 2003, MNRAS, 341, 1290 
Hedrosa, R. P., Abia, C., Busso, M., et al. 2013, ApJ, 768, L11

Hernanz, M., José, J., \& Coc, A. 2001, in Cosmic evolution, eds. E. VangioniFlam, R. Ferlet, \& M. Lemoine, 149

Hurley, J. R., Tout, C. A., \& Pols, O. R. 2002, MNRAS, 329, 897

Iben, I., \& Renzini, A. 1983, ARA\&A, 21, 271

Izumiura, H., Noguchi, K., Aoki, W., et al. 2008, ApJ, 682, 499

Izzard, R. G., Tout, C. A., Karakas, A. I., \& Pols, O. R. 2004, MNRAS, 350, 407

Izzard, R. G., Dray, L. M., Karakas, A. I., Lugaro, M., \& Tout, C. A. 2006, A\&A, 460,565

Izzard, R. G., Jeffery, C. S., \& Lattanzio, J. 2007, A\&A, 470, 661

Izzard, R. G., Glebbeek, E., Stancliffe, R. J., \& Pols, O. R. 2009, A\&A, 508, 1359

Jorissen, A., Van Eck, S., Mayor, M., \& Udry, S. 1998, A\&A, 332, 877

Jose, J., \& Hernanz, M. 1998, ApJ, 494, 680

José, J., Hernanz, M., Amari, S., Lodders, K., \& Zinner, E. 2004, ApJ, 612, 414

Kroupa, P., Tout, C., \& Gilmore, G. 1993, MNRAS, 262, 545

Lambert, D. L., Smith, V. V., \& Hinkle, K. H. 1990, AJ, 99, 1612

Lloyd Evans, T. 1991, MNRAS, 249, 409

Marks, P. B., Sarna, M. J., \& Prialnik, D. 1997, MNRAS, 290, 283

Morgan, D. H., Cannon, R. D., Hatzidimitriou, D., \& Croke, B. F. W. 2003, MNRAS, 341, 534

Nauenberg, M. 1972, ApJ, 175, 417

Nelson, L. A., MacCannell, K. A., \& Dubeau, E. 2004, ApJ, 602, 938

Nomoto, K., Saio, H., Kato, M., \& Hachisu, I. 2007, ApJ, 663, 1269

Ohnaka, K., \& Tsuji, T. 1999, A\&A, 345, 233

Ohnaka, K., Izumiura, H., Leinert, C., et al. 2008, A\&A, 490, 173

Paczynski, B. 1976, in Structure and Evolution of Close Binary Systems, eds.

P. Eggleton, S. Mitton, \& J. Whelan, IAU Symp., 73, 75
Paxton, B., Cantiello, M., Arras, P., et al. 2013, ApJS, 208, 4

Politano, M., van der Sluys, M., Taam, R. E., \& Willems, B. 2010, ApJ, 720, 1752

Pylyser, E., \& Savonije, G. J. 1988, A\&A, 191, 57

Romano, D., \& Matteucci, F. 2005, Nucl. Phys. A, 758, 328

Scott, A. D., Rawlings, J. M. C., Krautter, J., \& Evans, A. 1994, MNRAS, 268, 749

Shafter, A. W. 2002, in Classical Nova Explosions, eds. M. Hernanz, \& J. José, AIP Conf. Ser., 637, 462

Stehle, R., \& Ritter, H. 1999, MNRAS, 309, 245

Straniero, O., Gallino, R., Busso, M., et al. 1995, ApJ, 440, L85

Utsumi, K. 1985, in Cool Stars with Excesses of Heavy Elements, eds. M. Jaschek \& P. C. Keenan, Astrophys. Space Sci. Lib., 114, 243

Vanture, A. D. 1992, AJ, 103, 2035

Wallerstein, G., \& Knapp, G. R. 1998, ARA\&A, 36, 369

Warner, B. 1995, Cataclysmic variable stars., 28

Westerlund, B. E. 1997, The Magellanic Clouds (Cambridge: Cambridge Univ. Press)

Wheeler, J. C. 1992, in Evolutionary Processes in Interacting Binary Stars, eds. Y. Kondo, R. Sistero, \& R. S. Polidan, IAU Symp., 151, 225

Whelan, J., \& Iben, J. I. 1973, ApJ, 186, 1007

Wood, P. R., Bessell, M. S., \& Fox, M. W. 1983, ApJ, 272, 99

Yaron, O., Prialnik, D., Shara, M. M., \& Kovetz, A. 2005, ApJ, 623, 398

Yungelson, L., Livio, M., Tutukov, A., \& Kenyon, S. J. 1995, ApJ, 447, 656

Zhang, X., \& Jeffery, C. S. 2013, MNRAS, 430, 2113

Zorotovic, M., Schreiber, M. R., Gänsicke, B. T., \& Nebot Gómez-Morán, A. 2010, A\&A, 520, A86

Pages 12 to 13 are available in the electronic edition of the journal at http://www . aanda. org 


\section{Appendix A: Constraining CE ejection efficiency}

Our choice of the CE ejection efficiency parameter $\alpha_{\mathrm{CE}}$ is based on the following population synthesis estimate for the Galactic nova rate (per year) which is constrained by observations (Shafter 2002). In the population synthesis algorithm for counting novae, the total frequency $\left(v_{\text {tot }}\right)$ of nova-explosions (per year) for $Z=0.02$ and a constant star formation rate, $S=7.086 \mathrm{yr}^{-1}$ (following the prescription of Hurley et al. 2002), is estimated from,

$v_{\mathrm{tot}}=\sum_{i} S \times \delta p_{i} \times\left(\frac{\delta t_{\mathrm{nova}}}{\tau_{\mathrm{rec}}}\right)$,

with the nova recurrence time $\tau_{\text {rec }}$ for a binary system calculated for every timestep $\delta \mathrm{t}_{\text {nova }}$ of its evolution in a nova-phase from,

$\tau_{\mathrm{rec}}=\frac{\Delta M_{\mathrm{WD}}^{\mathrm{crit}}}{\dot{M}}$

using the expression of Yungelson et al. (1995) for the critical ignition mass $\Delta M_{\mathrm{WD}}^{\text {crit }}$,

$\Delta M_{\mathrm{WD}}^{\mathrm{crit}}=2 \times 10^{-6}\left(\frac{M_{\mathrm{WD}}}{R_{\mathrm{WD}}^{4}}\right)^{0.8}$

The WD radius $R_{\mathrm{WD}}$ is determined according to the formula of Nauenberg (1972),

$R_{\mathrm{WD}}=0.0112 \sqrt{\left(\frac{M_{\mathrm{Ch}}}{M_{\mathrm{WD}}}\right)^{\frac{2}{3}}-\left(\frac{M_{\mathrm{Ch}}}{M_{\mathrm{WD}}}\right)^{-\frac{2}{3}}}$,

where $M_{\mathrm{Ch}}=1.43 \mathrm{M}_{\odot}$ is the Chandrasekhar mass.

\section{A.1. Galactic nova rate}

With the above prescription, the total rate of novae in the Galaxy $(Z=0.02)$ is calculated using binary_c/nucsyn as,

$R_{\text {nova }}=f_{\text {bin }} \times v_{\text {tot }}$,

assuming a constant binary fraction $f_{\text {bin }}=0.5$ (Duquennoy \& Mayor 1991). As shown in Table A.1, our estimate of the Galactic nova rate is consistent with existing estimates of $30 \pm 10 \mathrm{yr}^{-1}$ (Shafter 2002) within a factor of $\sim 2-3$, being closer to the observed rate for values of $\alpha_{\mathrm{CE}}<0.5$, and decreasing with higher values of $\alpha_{\mathrm{CE}}$.

Figure A.1 further illustrates the sensitivity of the nova rate to the choice of $\alpha_{\mathrm{CE}}$. The nova rate decreases sharply at higher WD masses and for more efficient CE ejection. This is expected because the final separation after the CE phase increases with $\alpha_{\mathrm{CE}}$, so that for a higher $\alpha_{\mathrm{CE}}$, the post-CE binary is too wide for the secondary to evolve to fill its Roche lobe. Because of shorter recurrence times for massive WDs which lead to more frequent outbursts, the total nova rate drops sharply with increasing $\alpha_{\mathrm{CE}}(0.2$ to 0.5$)$. Nelson et al. (2004) also find an increase in the nova rate by a factor of $\sim 2-3$ for lower CE ejection efficiency. For $\alpha_{\mathrm{CE}}<0.2$, the nova rate decreases as more systems merge following the CE phase that leads to the WD-MS binary.
Table A.1. Estimated values of the Galactic nova rate, $R_{\text {nova }}$, average WD-mass in nova systems, $\left\langle M_{\mathrm{WD}}\right\rangle$, and fraction of WD binaries leading to novae, $\alpha$ (according to Eq. (A.6)), as a function of the CE ejection efficiency $\alpha_{\mathrm{CE}}$.

\begin{tabular}{cccc}
\hline \hline$\alpha_{\mathrm{CE}}$ & $R_{\mathrm{nova}}=f_{\text {bin }} \times v_{\text {tot }}\left(\mathrm{yr}^{-1}\right)$ & $\alpha$ & $\left\langle M_{\mathrm{WD}}\right\rangle$ \\
\hline 0.1 & 26.5 & 0.021 & 0.92 \\
0.2 & 49 & 0.021 & 1.07 \\
0.3 & 29.3 & 0.02 & 0.995 \\
0.4 & 18.6 & 0.02 & 0.92 \\
0.5 & 13.6 & 0.019 & 0.82 \\
1.0 & 10.9 & 0.02 & 0.77 \\
\hline
\end{tabular}

Notes. The frequency of novae, $v_{\text {tot }}$, is calculated according to Eq. (A.1) and a constant binary fraction $f_{\text {bin }}=0.5$ is used to estimate $R_{\text {nova }}$ according to Eq. (A.5).

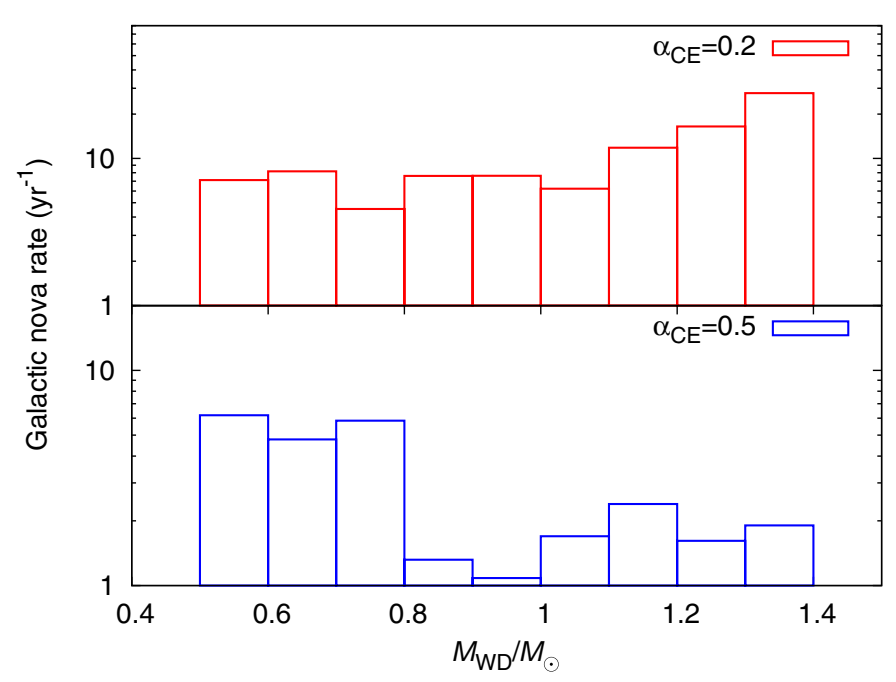

Fig. A.1. Histograms of predicted Galactic nova rate as function of WD mass, $M_{\mathrm{WD}}$ per $0.1 M_{\odot}$ bin, for $\alpha_{\mathrm{CE}}=0.2$ (upper panel) and 0.5 (lower panel). The total rate of novae in the Galaxy $R_{\text {nova }}$ is the sum of the contributions from all $M_{\mathrm{WD}}$ bins.

\section{A.2. Additional tests for nova binaries}

The fraction, $\alpha$, of binary systems hosting WDs that lead to novae is also estimated as,

$R_{\text {nova }}=\alpha R_{\mathrm{WD}}^{\mathrm{bin}}$

where $R_{\mathrm{WD}}^{\mathrm{bin}}$ is the birth rate of WDs in binary systems. Also, the frequency averaged WD mass $\left(\left\langle M_{\mathrm{WD}}\right\rangle\right.$ in $\left.M_{\odot}\right)$ is calculated for different choices of $\alpha_{\mathrm{CE}}(<1)$. As shown in Table A.1, our estimates for this fraction $\alpha$ (defined by Eq. (A.6)) is close to the value of 0.02 used by Romano \& Matteucci (2005) to match the observed nucleosynthesis yields for Galactic novae. The averaged (by nova frequency) WD mass $\left\langle M_{\mathrm{WD}}\right\rangle$ is also in agreement with the values in the range of $0.8-1.1 M_{\odot}$ as obtained by Nelson et al. (2004).

\section{Appendix B: HBB J-stars: single stars vs. binary mergers}

If mass ratio $q$ exceeds a critical value $q_{\text {crit }}$ (as defined in Hurley et al. 2002), RLOF from a GB star to a lower mass MS 
companion is dynamically unstable and mass transfer leads to a CE phase in which the system can merge depending on the initial separation. For such a Case-B RLOF merger, the MS star is absorbed into the envelope and the core-mass of the merger is determined by the core of the GB donor star at the onset of the $\mathrm{CE}$ phase. Consequently, following the $\mathrm{CE}$ phase, the merged star has a lower core mass on the GB compared to a single star of same total mass. Because the core mass at the base of the AGB phase in our synthetic models depends on the core mass at the base of the GB (Hurley et al. 2002), such mergers ascend the AGB with lower core masses than single AGB stars of similar total masses. Consequently they live longer on the AGB and, for total masses higher than about $4 M_{\odot}$ for which HBB occurs along with TDU, we predict such mergers also behave as J-stars for a longer phase on the AGB as compared to single HBB AGB stars. Figure B. 1 shows an example of the J-star phase for such a binary merger that lasts for about $1 \mathrm{Myr}$ compared to the much shorter span of about 0.1 Myr for an equivalent single star. The binary system initially consists of a $3.1 M_{\odot}$ primary star which overflows its Roche lobe on the GB with a core mass of $0.45 M_{\odot}$ that subsequently forms the core of the merged star following a CE phase with the $1.3 M_{\odot}(\mathrm{MS})$ secondary absorbed into the giant envelope. Thus, the merger core (and envelope) mass is significantly different from a single star of similar total mass, and consequently the core mass can only grow to $0.47 M_{\odot}$ at the end of the GB - significantly lower than the expected core mass $\left(\sim 0.73 M_{\odot}\right)$ of a corresponding single star. This in turn leads to a lower core mass at the start of the TPAGB for the binary merger and consequently it evolves for a longer phase with TDU because of which its surface $\mathrm{C} / \mathrm{O}$ exceeds 1 , and $\mathrm{HBB}$ that decreases the isotopic ratios of ${ }^{12} \mathrm{C} /{ }^{13} \mathrm{C}$ and ${ }^{12} \mathrm{C} /{ }^{14} \mathrm{~N}$ on the stellar surface classifying it as a $\mathrm{J}$-star.
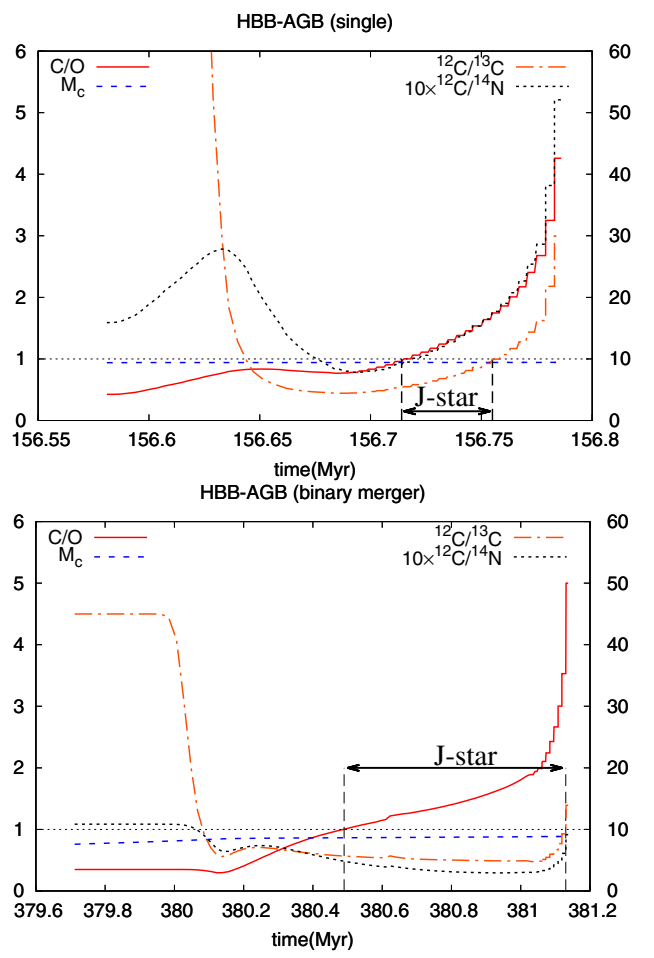

Fig. B.1. Evolutionary properties on the TPAGB for a single star (upper panel) and a GB-MS (Case-B RLOF) binary merger (lower panel) of similar total mass. The core mass $M_{\mathrm{c}}$ and surface $\mathrm{C} / \mathrm{O}$ are plotted on the left scale, along with surface isotopic ratios, ${ }^{12} \mathrm{C} /{ }^{13} \mathrm{C}$ and ${ }^{12} \mathrm{C} /{ }^{14} \mathrm{~N}$ (multiplied by a factor of 10) on the right scale. The dotted horizontal lines mark $\mathrm{C} / \mathrm{O}=1$ and ${ }^{12} \mathrm{C} /{ }^{13} \mathrm{C}=10$ while the dashed vertical lines mark the duration of the J-star phase. 